\title{
An Ultra-Wideband Single-Polarization-Single-Mode Terahertz Photonic Crystal Fiber
}

\author{
$1^{\text {st }}$ Tianyu Yang \\ Global Big Data Technolgoies \\ Centre \\ Universiy of Technology Sydney \\ Sydney, Australia \\ tianyu.yang@student.uts.edu.au
}

\author{
$2^{\text {nd }}$ Can Ding \\ Global Big Data Technolgoies \\ Centre \\ Universiy of Technology Sydney \\ Sydney, Australia \\ can.ding@uts.edu.au
}

\author{
$3^{\text {rd }}$ Richard W. Ziolkowski \\ Global Big Data Technolgoies \\ Centre \\ Universiy of Technology Sydney \\ Sydney, Australia \\ richard.ziolkowski@uts.edu.au
}

\author{
$4^{\text {th }}$ Y. Jay Guo \\ Global Big Data Technolgoies \\ Centre \\ Universiy of Technology Sydney \\ Sydney, Australia \\ jay.guo@uts.edu.au
}

\begin{abstract}
A terahertz (THz) photonic crystal fiber (PCF) with an ultra-wide bandwidth and single-polarization-singlemode (SPSM) operation is designed and analyzed. Two air slots are introduced in the core region and epsilon-near-zero (ENZ) material is deposited in four specific air holes in the cladding of the PCF. The design achieves significantly different electric (E)field distributions of the X-polarized (XP) and Y-polarized (YP) modes. The E-field components overlapping the ENZ material are attenuated because it is lossy. Gain material is then deposited in a rectangular slot in the core center to provide amplification of the E-field components overlapping this gain region. Changing the dimensions of the PCF modifies the amplification and attenuation rates to the wanted XP mode, the unwanted YP mode, and any unwanted higher order (HO) modes. The amplification of the wanted mode and the attenuation of the unwanted modes are maximized through optimization. The result is a PCF with an ultra-wide SPSM spectrum of $0.53 \mathrm{THz}$, from 1.00 to $1.53 \mathrm{THz}$. The minimum loss difference (MLD) across this bandwidth between the wanted mode and any unwanted modes is over $7.4 \mathrm{~dB} / \mathrm{cm}$. To the best of our knowledge, this is the widest SPSM bandwidth of a PCF fiber reported in THz regime.
\end{abstract}

Keywords-Guided wave modes, photonic crystal fiber, single polarization single mode, terahertz.

\section{INTRODUCTION}

To meet the ever-increasing capacity requirements of data transmission, terahertz $(\mathrm{THz})$ band systems are expected to be a core element in future generations of wireless communications [1]. The THz band, which is generally defined in the range from $0.1-10 \mathrm{THz}(30-3000 \mu \mathrm{m})$, has attracted enormous attention over the last decade. This is due to the fact that wide bands of unallocated spectrum are available that could be used for various applications, e.g., sensing, imaging, security, and communications [2]. THz waveguides play an important role in all $\mathrm{THz}$ systems. Among various $\mathrm{THz}$ waveguides, photonic crystal fibers (PCFs) are one of the most promising candidates for those applications due to their low transmission losses and flexible guiding characteristics [3],[4].

Polarization maintenance (PM) is one preferred or often required characteristic for $\mathrm{THz}$ waveguides since it can eliminate crosstalk and modal dispersion between the two fundamental modes propagating in them. The best way to realize PM is to use single-polarization-single-mode (SPSM) fibers $[5,6]$. While SPSM PCFs have been successfully deployed in the optical regime, only a few works have reported them in the $\mathrm{THz}$ regime, despite the increased demand for $\mathrm{THz}$ communications and sensor systems. SPSM PCFs require proper tailoring of the loss differences (LDs) between the wanted mode and the unwanted modes. Basically, all reported optical SPSM PCFs are based on engineering the confinement loss (CL) differences between the two fundamental modes. However, the effective material loss (EML) is dominant in the $\mathrm{THz}$ regime. Our previous work [6] introduced the first $\mathrm{THz}$ SMSP PCF based on emphasizing the EML engineering. While the achieved performances were reasonable, i.e., SPSM bandwidth $(\mathrm{BW})=0.31 \mathrm{THz}$ and minimum $\mathrm{LD}$ (MLD) values $>8.0 \mathrm{~dB} / \mathrm{cm}$, the design was quite preliminary. In this work, a new configuration is developed. By introducing air slots in the core region, the optimized SPSM PCF has a bandwidth of 0.53 $\mathrm{THz}$ and a MLD value $>7.4 \mathrm{~dB} / \mathrm{cm}$ over its entire operational band. Compared to the previous design, the bandwidth has been enhanced by $70 \%$ while the MLD is comparable.

\section{CONFIGURATION AND NUMERICAL ANALYSIS}

Fig. 1 shows the cross-sectional view of the developed PCF configuration. High resistivity silicon (HRS) [7] is adopted as the substrate. Five layers of triangularly distributed air hole arrays form the cladding. Four air holes surrounding the core region are selected to have lossy epsilon-near-zero (ENZ) material [8] shaped rings with a thickness of $t$ deposited in them. The four air holes augmented with these ENZ rings have the diameter $d_{2}$. The rest of the air holes have the diameter $d_{1}$. The distance between any two adjacent air holes is the lattice constant $\Lambda$. There are three horizontally arranged slots with different dimensions in the core area. The red slot is filled with the background substrate doped with gain material having the gain factor $\xi$; the other two slots are filled with air. The sizes of the air slots and the gain slot are $L_{a} \times W_{a}$ and $L_{g} \times W_{g}$, respectively. After optimization, the design parameters are: $\Lambda$ $=70 \mu \mathrm{m}, d_{1}=0.95 \Lambda, d_{2}=0.83 \Lambda, L_{a}=L_{g}=0.5 \Lambda, W_{a}=0.14 \Lambda$, $W_{g}=0.08 \Lambda, D=0.456 \Lambda, \xi=-0.03$, and $t=0.18 \Lambda$.

Fig. 2 shows the magnitudes of the E-field distribution of the X-polarized (XP), the Y-polarized (YP), and the lowest loss higher-order (HO) modes. It is observed that the E-field of the XP mode is highly localized in the core area while that of the 


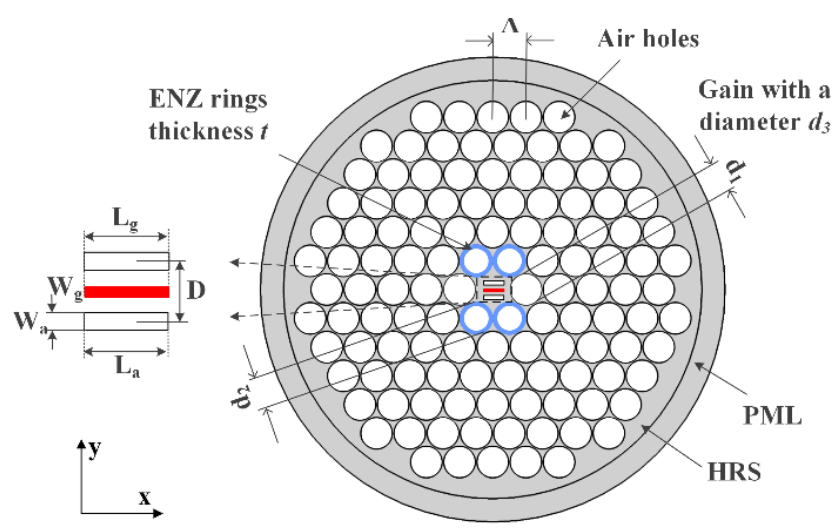

Fig. 1. Cross-section view of the reported SPSM PCF.
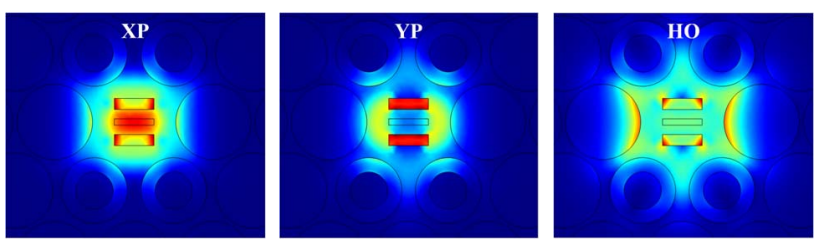

Fig. 2. E-field distribution of the XP, YP, and $\mathrm{HO}$ modes at $1.2 \mathrm{THz}$.

YP mode is concentrated in the air slots. Therefore, the gainfilled slot located in the core center will produce a much larger amplification of the XP mode than that of the YP mode. Compared to the XP mode, the YP mode has a larger E-field intensity in the lossy ENZ rings. It thus experiences a higher attenuation as it propagates. Therefore, it can be deduced that the YP mode will have a much higher loss than the XP mode. On the other hand, the $\mathrm{HO}$ mode is seen to be more concentrated near the lossy ENZ rings and less so in the core region. Consequently, it will also experience smaller amplification from the gain area and a larger attenuation rate from the ENZ rings in comparison to the XP mode. These features lead to the desired large LD values between the wanted XP mode and all of the other unwanted modes.

\section{RESULTS}

The optimal simulated total loss (TL) values, i.e., the sum of CL and EML values, of the different modes as functions of the frequency are shown in Fig. 3(a). It is noticed that the TLs of all the modes generally decrease as the frequency increases. Also note that the TL of the XP mode attains negative values after $1.00 \mathrm{THz}$, while the TLs of all of the other modes remain positive until $1.53 \mathrm{THz}$. Within this range, the XP mode would be amplified and all of the other modes would be attenuated. This result means that after propagating in the PCF for a certain distance, only the XP mode would remain, i.e., singlepolarization-single-mode operation is achieved. Therefore, this frequency range is defined as the working band. The corresponding LD values are plotted in Fig. 3(b). It is further noticed that the LDs between the YP and XP modes have their lowest values before $1.43 \mathrm{THz}$. After that frequency the LDs between the HO and XP modes exhibit the lowest values. The MLD value across the whole SPSM bandwidth is determined by the LD value around $1.53 \mathrm{THz}$, which is $7.4 \mathrm{~dB} / \mathrm{cm}$. Compared to our previous work [6], the SPSM bandwidth is widened by $71 \%$ with only a $7.5 \%$ sacrifice of the MLD value.

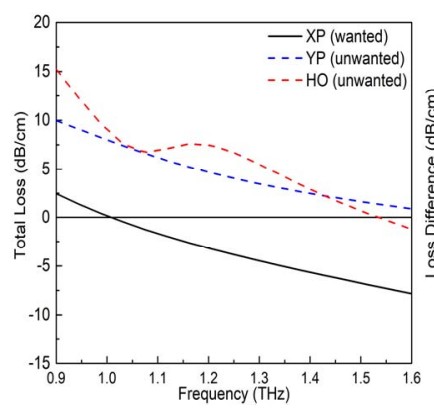

(a)

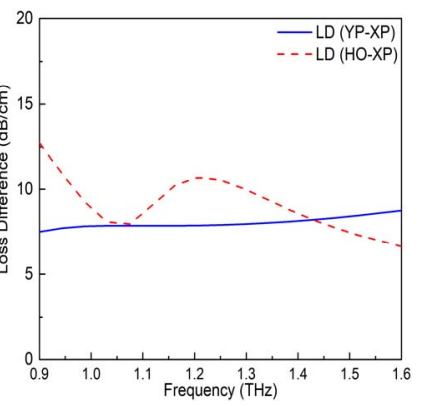

(b)
Fig. 3. Simulated TL values of the XP, YP, and HO modes of the PCF as functions of the source frequency. (b) The corresponding $L D$ values between the wanted (XP) and unwanted (YP, HO) modes over the operational band.

\section{CONCLUSION}

A THz SPSM PCF design was developed. It achieves an ultra-wide bandwidth over which SPSM propagation is supported with large MLD values between the wanted (XP) and unwanted (YP and $\mathrm{HO}$ ) modes. The significant improvements of the SPSM bandwidth and MLD values were attributed to a custom-designed core region in which three rectangular slots were introduced, the middle one being filled with a gain-doped substrate, and to lossy ENZ material introduced as rings into four selected air holes surrounding the core area. Extensive parametric studies were conducted to explain the resulting behaviors of the wanted and unwanted modes and to obtain the optimized structural parameters. The operating mechanisms were illustrated with this optimized design. With such a wide SPSM bandwidth and large MLD performance, the reported PCF is a competitive candidate for a variety of $\mathrm{THz}$ wave guiding applications requiring polarization purity.

\section{REFERENCES}

[1] I. F. Akyildiz, J. M. Jornet, C. Han, "Terahertz band: Next frontier for wireless communications," Phys. Com., vol. 12, pp. 16-32, 2014.

[2] A. Redo-Sanchez and X.-C. Zhang, "Terahertz science and technology trends," IEEE J. Sel. Top. Quantum Electron., vol. 14, pp. 260-269, 2008.

[3] T. Yang, E. Wang, H. Jiang, Z. Hu, and K. Xie, "High birefringence photonic crystal fiber with high nonlinearity and low confinement loss," Opt. Express, vol. 23, pp. 8329-8337, 2015.

[4] S. Atakaramians, S. Afshar V, H. Ebendorff-Heidepriem, M. Nagel, B. M. Fischer, D. Abbott, and T. M. Monro, "THz porous fibers: design, fabrication and experimental characterization," Opt. Express, vol. 17, pp. 14053-15062, 2009.

[5] Y. Huo, F. Fan, H. Zhang, X. Wang, and S. Chang, "Terahertz singlepolarization single-mode hollow-core fiber based on index-matching coupling,” IEEE Photon. Techno. Lett., vol. 24, pp. 637-639, 2012.

[6] T.-Y. Yang, C. Ding, R. W. Ziolkowski, Y. J. Guo, "A terahertz (THz) single-polarization-single-mode (SPSM) photonic crystal fiber (PCF)," Materials, vol. 12, pp. 2442, 2019.

[7] J. Dai, J. Q. Zhang, W. L. Zhang, and D. Grischkowsky, "Terahertz time-domain spectroscopy characterization of the far-infrared absorption and index of refraction of high-resistivity, float-zone silicon," Opt. Soc. Am. B, vol. 21, pp. 1379-1386, 2004.

[8] G. V. Naik, V. M. Shalaev, and A. Boltasseva, "Alternative plasmonic materials: beyond gold and silver," Adv. Mater., vol. 25, pp. 3264-3294, 2013. 